\title{
Muscle activity and head kinematics in unconstrained movements in subjects with chronic neck pain; cervical motor dysfunction or low exertion motor output?
}

\author{
Harald Vikne ${ }^{1 *}$, Eva Sigrid Bakke ${ }^{1}$, Knut Liestøl ${ }^{2}$, Stian R Engen ${ }^{1}$ and Nina Vøllestad ${ }^{1}$
}

\begin{abstract}
Background: Chronic neck pain after whiplash associated disorders (WAD) may lead to reduced displacement and peak velocity of neck movements. Dynamic neck movements in people with chronic WAD are also reported to display altered movement patterns such as increased irregularity, which is suggested to signify impaired motor control. As movement irregularity is strongly related to the velocity and displacement of movement, we wanted to examine whether the increased irregularity in chronic WAD could be accounted for by these factors.

Methods: Head movements were completed in four directions in the sagittal plane at three speeds; slow (S), preferred (P) and maximum (M) in 15 men and women with chronic WAD and 15 healthy, sex and age-matched control participants. Head kinematics and measures of movement smoothness and symmetry were calculated from position data. Surface electromyography (EMG) was recorded bilaterally from the sternocleidomastoid and splenius muscles and the root mean square (rms) EMG amplitude for the accelerative and decelerative phases of movement were analyzed.
\end{abstract}

Results: The groups differed significantly with regard to movement velocity, acceleration, displacement, smoothness and rmsEMG amplitude in agonist and antagonist muscles for a series of comparisons across the test conditions (range $17-121 \%$, all p-values $<0.05$ ). The group differences in peak movement velocity and acceleration persisted after controlling for movement displacement. Controlling for differences between the groups in displacement and velocity abolished the difference in measures of movement smoothness and rmsEMG amplitude.

Conclusions: Simple, unconstrained head movements in participants with chronic WAD are accomplished with reduced velocity and displacement, but with normal muscle activation levels and movement patterns for a given velocity and displacement. We suggest that while reductions in movement velocity and displacement are robust changes and may be of clinical importance in chronic WAD, movement smoothness of unconstrained head movements is not.

Keywords: Whiplash associated disorder, Persistent neck pain, Movement kinematics, Electromyography, Neck muscles, Movement smoothness

\footnotetext{
* Correspondence: harald.vikne@medisin.uio.no

1 Department of Health Sciences, Institute of Health and Society, University of Oslo, P.O. Box 1089, Blindern, NO-0317 Oslo, Norway

Full list of author information is available at the end of the article
} 


\section{Background}

People having long-term musculoskeletal neck pain after motor vehicle accidents (Whiplash associated disorders WAD) may have pronounced disability that affects daily living [1]. One of the most prominent clinical manifestations in persons with long-term WAD is cervical motor dysfunction [2], signified by altered neck muscle activation and reduced peak motor output. For example, measures of peak performance such as low load isometric endurance and maximum voluntary isometric contraction force of neck- and shoulder girdle muscles are known to be reduced in chronic WAD [3-9]. For relatively unconstrained dynamic head movements, kinematic performance variables such as peak velocity are also lower than in healthy participants $[10,11]$ and the peak head movement displacement is typically reduced in people with chronic WAD [10-17]. Collectively, these alterations reduce the functional capacity of the head and neck in people with chronic WAD.

The causes for the reductions in peak kinematic performance variables in chronic WAD are less clearly understood. It has been shown that the activation of neck muscles is altered in chronic WAD as compared with healthy subjects $[5,18]$ and such changes may potentially affect the head kinematics. However, these results were obtained from studies of isometric neck muscle contractions at low to moderate muscle forces $[5,18]$. It is therefore possible that these observations are not directly applicable to dynamic head movements. In a study of movement kinematics in chronic WAD and control participants, Sjölander [19] found no difference in peak movement velocity when taking movement displacement into consideration. Because of the close relationship between the kinematic parameters of displacement and peak velocity [20], it is therefore possible that reductions in head displacement may contribute to the reductions in peak head movement velocity in chronic WAD.

In addition to measures of peak performance, the regularity or smoothness of neck movements in people with chronic neck pain are reported to be reduced [11,15,19,21]. Smooth movements are characterized by approximately bell-shaped and unimodal velocity profiles [22], while movements of reduced smoothness exhibit multi-peaked, irregular velocity profiles containing a series of accelerative and decelerative phases. Such irregular movement patterns have been suggested to be a consequence of motor control disturbance in people with persistent WAD $[11,19]$. In a previous study, the irregularity of movement was shown to be strongly related to both the movement velocity and displacement across a series of different head movements in healthy participants [23]. Since it is known that people with chronic WAD perform with both lower movement velocity and less displacement compared with controls, it raises the question of whether the reduced smoothness of movements observed in people with chronic WAD may simply be caused by altered movement velocity and displacement and not altered movement control strategies.

In this study we compared head kinematics and muscle activation in relatively unconstrained neck movements at three different speeds in participants with and without chronic WAD. In addition comparisons were made taking both movement velocity and displacement into consideration.

\section{Methods \\ Participants}

We examined 15 patients (six men and nine women) suffering from chronic WAD (> 6 months), classified as grade 2 according to the Quebec Task Force classification [24] and which started less than 72 hours after the motor vehicle accident. In addition, six men and nine women matched with the WAD group for sex and age ( \pm 5 years) served as controls. The following exclusion criteria were used: WAD grade 3-4, pregnancy, age $\leq 18$ or $\geq 60$ years, unsettled insurance claims, systemic inflammatory diseases, neurological disorders, tremor, regular usage of analgesics and strongly reduced vision/ blindness or auditory defects. All patients were recruited from a local rehabilitation clinic and examined by a specialist in physical medicine or neurology and a manual therapist before inclusion. Descriptive data for the participant groups are given in Table 1 . The study was approved by the Regional Committee for Medical and Health Research Ethics, and all participants signed an informed consent form for participation in the study in accordance with the Helsinki declaration.

\section{Overview and procedures}

In this study we examined the movement performance of unconstrained head movements in the sagittal plane at three different speeds in participants with and without chronic WAD. Head movement performance was assessed with respect to displacement, velocity and acceleration and measures of movement smoothness and symmetry. Neck muscle activity was measured by means of surface electromyography (EMG) of agonist and antagonist muscles. Descriptive measures of anthropometry and overall strength were taken as they may affect the outcome variables in the study. All experiments were performed in a standardized laboratory setting. All participants completed one separate training session in order to familiarize themselves with the testing procedures 1-2 weeks in advance of the experiment. Tests were completed in the following order: 1) maximum handgrip strength, 2) evaluation of pain intensity, 3) tests of head movements and 4) re-evaluation of pain intensity. Participants were given pauses ad libitum. 
Table 1 Descriptive data for the chronic WAD and control groups (six men and nine women in each group)

\begin{tabular}{lll}
\hline Variable & WAD & Control \\
\hline Age (yrs) & $40.1(8.7)$ & $38.7(8.8)$ \\
Height (cm) & $170.5(8.5)$ & $173.2(7.6)$ \\
Weight (kg) & $78.3(13)$ & $75.8(13.9)$ \\
BMl (kg/m²) & $26.9(4.2)$ & $25.1(3.2)$ \\
Head mass (kg) & $4.33(0.34)$ & $4.53(0.30)$ \\
Hand grip strength, dominant (kg) & $45.3(11.4)$ & $50.1(12.6)$ \\
& $41.8(11.8)$ & $47.3(12.1)$ \\
SF-36, PCS (0-100) & $33.4(9.7)^{* *}$ & $54.4(5.0)(\mathrm{n}=14)$ \\
\multicolumn{1}{c}{ MCS (0-100) } & $45.3(15.0)^{*}$ & $55.2(4.8)(\mathrm{n}=14)$ \\
Duration of symptoms (months) & $22(98)$ & - \\
Pain intensity, pre-test (1-10) & $3.1(1.4)$ & - \\
\multicolumn{1}{c}{ post-test (1-10) } & $5.6(2.0) \#$ & - \\
NDI (0-50) & $21.7(5.6)$ & - \\
FABQ, W (0-42) & $22.3(10.2)$ & - \\
\multicolumn{1}{c}{ PA (0-24) } & $10.5(4.5)$ & - \\
\hline
\end{tabular}

Results are average (SD). For the duration of symptoms the results are median (interquartile range). Statistically significant difference from the control group; ${ }^{*} p<0.05$, $* * 0<0.0005$. Statistically significant difference from the pre-value within group; \# $p<0.0005$.

Abbreviations; BMI body mass index, SF-36 short form-36, PCS physical component summary, MCS mental component summary, NDI neck disability index $F A B Q$ fear avoidance beliefs questionnaire, $W$ work, $P A$ physical activity.

\section{Descriptive data}

\section{Anthropometrics and grip strength}

The participants' body height $(\mathrm{cm})$ and weight $(\mathrm{kg})$ were measured and the body mass index $\left(\mathrm{kg} / \mathrm{m}^{2}\right)$ calculated. Head volume was measured for men and women as described by McConville [25] and Young [26], respectively and a density of $1.05 \mathrm{~kg} / \mathrm{l}$ [27] was used to estimate the head mass. As a measure of overall muscle strength $[28,29]$, hand grip strength was tested on a hand dynamometer (Model 78010, Lafayette Instruments) adjusted individually. The base rested on the first metacarpal and the bar on the second to fifth medial phalanx. Participants were told to squeeze as hard as possible and to maintain the force for three to four seconds. Each hand was tested two to three times (60 s inter-test pause) and the highest value was used in further analysis.

\section{Self-reported questionnaires}

A numerical rating scale (1-10) was used to assess subjective pain intensity in the neck and head region at the time of measurement, where 1 represents absence of pain and 10 the worst imaginable pain. Participants rated the pain intensity in the head and neck immediately before and after completion of the neck movement testing. The neck disability index (NDI) was used as a measure of physical disability due to neck pain [30]. Fear of movement and of movement-related pain in work and physical activity in general was measured using the fear avoidance beliefs questionnaires (FABQ) work and physical activity subscales [31]. In line with previous studies in people with neck pain [32], we modified the FABQ by replacing the word back with neck. The health-related quality of life was assessed by the generic Short-Form Health Survey 36 (SF-36), version 1 [33]. The questionnaire's mental and physical component summary measures were calculated using Norwegian normative values [34], where the population norm score is defined as $50 \pm 10$ (SD) for both scales.

\section{Head movements \\ Movement directions}

Four head movements were completed in a custom designed chair as previously reported [23]. While sitting the participants were instructed to position themselves in their individual resting position with respect to their head when looking straight forward at a wall that was approximately $120 \mathrm{~cm}$ in front of them. This was defined as their neutral head position (NP). When sitting in this position, a $15 \mathrm{~mm}$ diameter dark blue dot was applied at the participant's individual focus point on the wall as an individual reference for the NP. The participants completed four head movements in the sagittal plane, each corresponding to approximately half of their full range of motion: forward flexion from NP (FFN), extension back to NP (EBN), extension from NP (EFN) and flexion back to NP (FBN). Participants were asked to move their head and neck as far as possible when starting from the NP and to stop at NP when starting from the fully flexed or extended position. The order of the direction of movements was randomized for each participant. Examples of two movement directions are shown in the top of Figure 1.

\section{Movement speeds}

The participants were tested in three different speed conditions. First, the participants were instructed to complete all movement directions in a pace corresponding to what they perceived as their normal speed, which was termed preferred speed (P). Thereafter they were instructed to move at about half of their preferred speed, termed slow speed (S) and finally with their maximum speed (M). To put as little constraint on the movements as possible, the participants were not given any feedback on their performance during testing. The participants were allowed to practice the movement directions and speed conditions before the test started and usually 2-4 trials were performed. The participants completed 3 trials per speed condition for each direction and these were averaged for further analysis. All trials were accepted, except if the participants expressed that the movements deviated from what they had intended to do, then retrials were performed. 


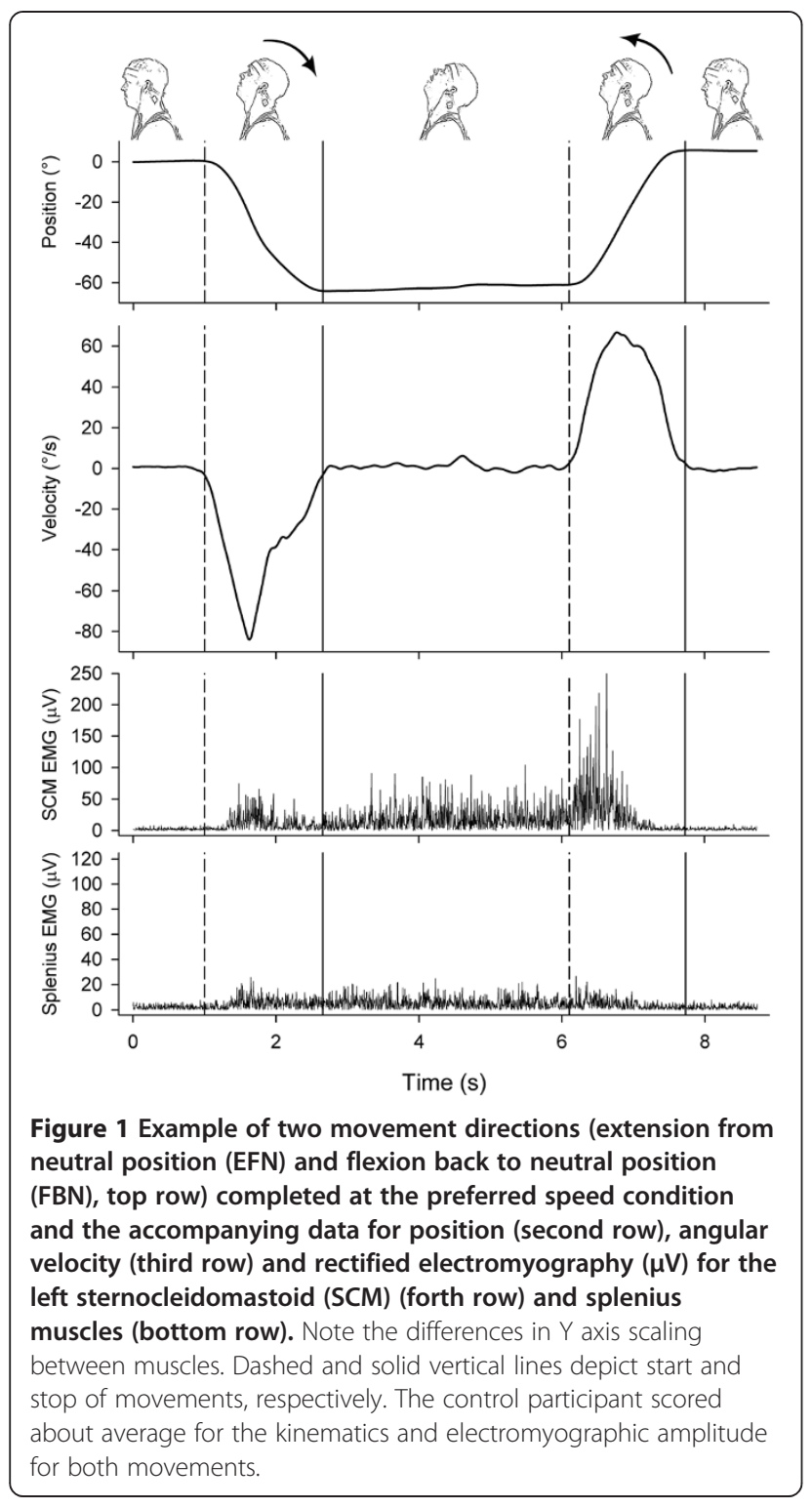

\section{Kinematics}

\section{Data sampling and analysis}

Position data were sampled using an electromagnetic motion tracker (Liberty, Polhemus Inc.) at $240 \mathrm{~Hz}$ as previously described in detail [23]. The signals, analyzed off-line in MatLab, were filtered using a quintic Woltring spline with a cutoff frequency of $6 \mathrm{~Hz}$. The quintic spline additionally defines the higher order derivatives (velocity, acceleration and jerk). Movement onset and offset were defined to be $4 \%$ of the peak angular velocity [23]. If the signal fluctuated across this $4 \%$ threshold, the final crossing was used for the offset. Movement duration and displacement were defined as the time and angular position difference between the movement onset and offset, respectively. The overall smoothness of the movement was calculated as the normalized jerk cost (NJC) according to
Teulings [35]. To further examine the regularity of the movement, the number of submovements was counted as described by Ketcham [36]. Movement symmetry for movements consisting of one submovement was measured by the velocity profile symmetry index [37], taken as the time to peak velocity divided by total movement time. Values less than or above 0.5 indicate asymmetry in the velocity profile. For movements consisting of more than one submovement, the spatial occurrence of the submovements was calculated as the relative number of submovements started in each of the two movement halves. The reliability of this setup and kinematic outcome measures have been previously examined and shown to be acceptable [23].

\section{Electromyography}

\section{Muscles and sensor placement}

Electromyographic signals were sampled bilaterally from the sternocleidomastoid (SCM) and the splenius muscles. The signals were detected and pre-amplified $10 \mathrm{x}$ using single differential active surface sensors consisting of two parallel $10 \times 1 \mathrm{~mm}$ silver electrode bars (DE-2.1, Delsys Inc.). The sensor placement on the SCM muscle were marked on the skin using published suggestions [38], then examined by ultrasound imaging using a $10 \mathrm{MHz}, 5 \mathrm{~cm}$ linear array probe (Vingmed, General Electrics) and adjusted if necessary. After locations were established, the skin was first shaved and then firmly rubbed and washed with 70\% isopropyl alcohol using electrode prep pads and the electrodes fastened using double adhesive tape. A $50 \mathrm{~mm}$ diameter ground electrode was placed over the left olecranon. From now on the SCM will be referred to as agonist during flexion movements and antagonist during extension movements. The opposite will be done for the splenius muscle. See Additional files 1 and 2 for a more detailed description.

\section{Data sampling and analysis}

The pre-amplified signals were passed to a main amplifier (Bagnoli-16, Delsys), amplified 1000x, band-pass filtered between $20-450 \mathrm{~Hz}$ with a built-in analog filter, ADconverted (NI-DAQ 6220, National Instruments) and sampled at $1 \mathrm{KHz}$. EMG signals were offset-adjusted and the running root mean squares (rms) were calculated in window lengths of $50 \mathrm{~ms}$ with $49 \mathrm{~ms}$ window overlap using an EMG software package (EMGworks 3.7). Baseline EMG was subtracted from the reference- and movement rmsEMG signals. EMG epochs covering the entire movement as defined by the kinematic start and stop procedures $\pm 200 \mathrm{~ms}$ were subsequently normalized to the median rmsEMG accomplished at reference contractions (see Additional files 1 and 2 for descriptions) and further analyzed. A few trials containing large spiked artifacts 
were excluded. The signal obtained during movement was separated into two epochs; one beginning at the start of movement as defined above for the position data and ending at the time point of peak velocity was defined as the accelerative phase, and one epoch starting at peak velocity and ending at the stop of movement was defined as the decelerative phase. The signals of bilateral muscle pairs were averaged for further analysis. Due to very low EMG activity during the $\mathrm{S}$ and $\mathrm{P}$ speed conditions for the gravity-assisted movements (EBN and FFN), the EMG was analyzed for the movements completed against gravity, i.e. the flexion and extension back to neutral position (FBN and EBN).

\section{Statistics}

Graphical displays were used to assess the distribution of the data. After log-transformation of right-skewed distributions, the data were found to be approximately normally distributed. The WAD and the control group were compared using independent samples t-tests. Differences within the groups between speed test conditions within a given movement direction were examined using analysis of variance for repeated measures.

Possible effects of velocity and displacement on the NJC and number of submovements were also examined by comparing groups using general linear models with velocity and displacements as covariates. As both displacement and velocity affects the EMG amplitude [39], the rmsEMG data were compared using the same model and covariates. Since peak movement velocity is strongly related to the displacement of movement [20], we also compared groups for peak velocity and acceleration at the $\mathrm{M}$ speed conditions using displacement as a covariate.

To test for differences between groups in the spatial distribution of submovements and of the velocity profile symmetry index we used a mixed factor general linear model with participants as random factor. Bivariate correlations were performed using Pearson's correlation coefficient. The scores of NDI, FABQ and pain intensity were analyzed against movement displacement, peak velocity and acceleration and rmsEMG amplitude of the P and $M$ speed condition for all movement directions. Tests are two-sided and p-values less than 0.05 were considered statistically significant. Statistical analyses were performed using the SPSS 18 and JMP 9.0 statistical packages.

\section{Results}

\section{Group characteristics}

The chronic WAD group and the control group were not significantly different with respect to age, anthropometrics or grip strength (Table 1). The WAD group displayed statistically significantly lower values of both the physical and mental component summary scales of the SF-36 than the control group did (p-values $<0.05)$. According to the scale of Vernon [30], the mean absolute NDI score of 22 for the WAD group was in the upper part of the range (15-24) defining moderate physical disability due to neck pain. The fear avoidance levels of the WAD group related to physical activity and work were also moderate.

\section{Kinematics}

All movement variables for head and neck kinematics showed differences between participants with- and without chronic WAD and the detailed results are presented in Figure 2 and Table 2. In summary, the mean values for displacement were numerically lower for the WAD group compared to controls in all comparisons across all movement directions; in 8 of 12 cases the differences were statistically significant ( $p$-values $<0.05$ ). Similar results were also found for both peak and average velocity as 16 of 24 comparisons were significantly lower for the WAD group ( $\mathrm{p}$-values $<0.05$ ). The peak acceleration and deceleration were significantly lower in the WAD group in 14 of 24 cases ( $p$-values $<0.05$ ). Peak and average velocity and peak acceleration and deceleration were significantly lower in the WAD group compared to the control group for all movement directions for the $M$ speed condition ( $\mathrm{p}$-values $<0.01$ ). Also, for the preferred test speed, the peak and average velocity and peak acceleration and deceleration were lower in the WAD group for the EFN and FBN movement.

The differences between groups in peak velocity and acceleration at the $M$ speed conditions were also evident after using displacement as a covariate (p-values $<0.05$ ).

Mean values for NJC and number of submovements were numerically consistently higher in the WAD group than the control group (Table 2), although the large variation between individuals implied that the difference was significant $(\mathrm{p}<0.05)$ only for one and two test conditions, respectively. However, when displacement and velocity were used as covariates, no differences were found between groups for either NJC or number of submovements at any test velocity for any movement direction (Figure 3). We found no statistically significant differences between groups in the spatial distribution of submovements in either of the two movement halves (both $\mathrm{p}=0.91$ ); for the WAD group, $54 \pm 17 \%$ of the submovements started in the first half of the movement displacement compared to $57 \pm 18 \%$ in the control group. Nor did we find any significant difference in the velocity profile symmetry for the movements consisting of one submovement only $(\mathrm{p}=0.81$; Figure 4$)$. In summary, we detected no difference in either the smoothness or the symmetry of movement between the two groups. 


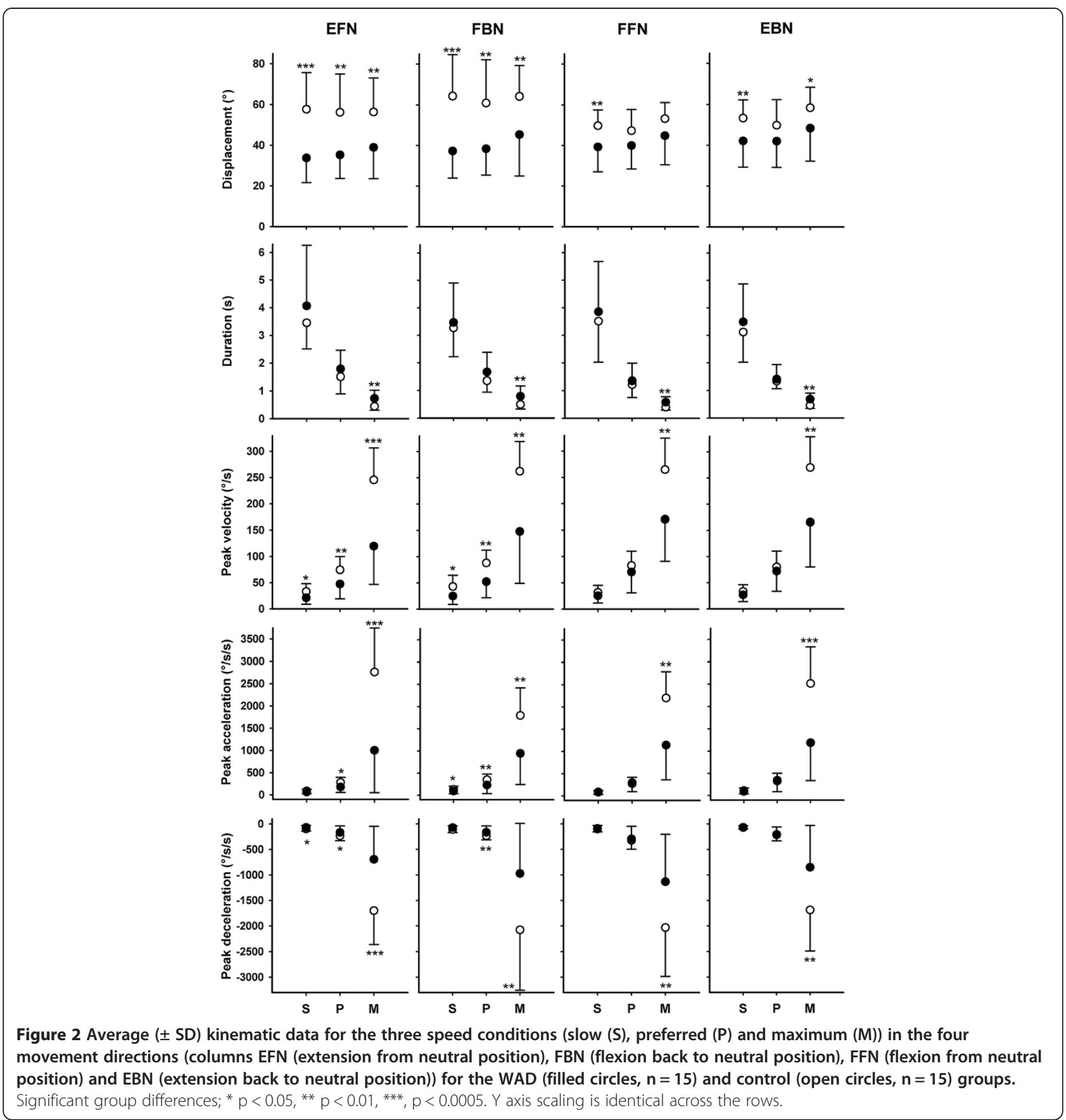

\section{Electromyography}

In the sitting position prior to testing, the absolute baseline rmsEMG amplitude $(\mu \mathrm{V})$ was not significantly different between groups for any muscle studied (Table 3, p-values $>0.25$ ). Similarly, we found no group differences in absolute rmsEMG amplitude $(\mu \mathrm{V})$ values during the reference contractions for the muscles ( $\mathrm{p}$-values $>0.11$ ).

There was an overall effect of increased movement velocity as assessed by the separate test speed conditions on both the agonistic and antagonistic muscle rmsEMG amplitude for the two phases of both movement directions analyzed (14 of 16 comparisons were statistically significant, $\mathrm{p}$-values $<0.05)$. For the acceleratory phase of the $M$ speed condition for all movement directions, the relative rmsEMG amplitude of both the agonistic and antagonistic muscles were significantly lower for the WAD group compared with the controls (p-values $<0.01$, Figure 5). For the decelerative phases of these movements, only the antagonistic muscles displayed lower amplitude for the WAD group (p-values $<0.01$ ). 
Table 2 Average (SD) angular velocity ( $\%$ s), normalized jerk cost (a.u.) and number of submovements for the three speed conditions in the four movement directions for the chronic WAD ( $n=15)$ and control groups $(n=15)$

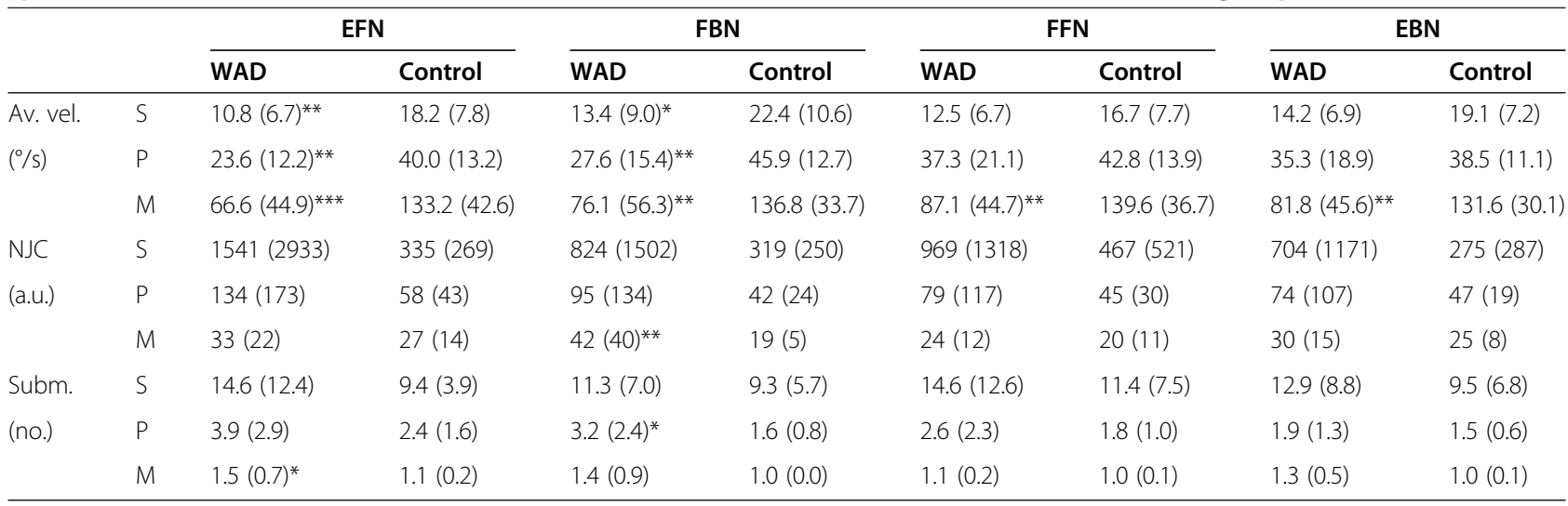

Statistically significant difference from the control group; ${ }^{*} p<0.05,{ }^{* *} p<0.01$.

Abbreviations; NJC normalized jerk cost, a.u. arbitrary units, Subm. submovement, no. number, Av.vel. average velocity, NP neutral head position, EFN extension from NP, FBN flexion back to NP, FFN flexion from NP, EBN extension back to NP, $S$ slow movement speed, $P$ preferred movement speed, $M$ maximum movement speed.

For the FBN direction, reduced muscle activity in the WAD group was also found for the accelerative phase at the $\mathrm{P}$ speed condition in both the agonist and antagonistic muscles and at the $S$ speed condition for the antagonistic muscles ( $\mathrm{p}$-values $<0.05$ ). No group differences were found for the EBN direction at either the $\mathrm{S}$ or $\mathrm{P}$ speed (p-values $>0.38$ ).

When the EMG data was compared between groups while controlling for velocity and displacement, all statistically significant differences between groups in rmsEMG amplitude for the agonist and the antagonist muscles vanished ( $p$-values $>0.18$ ) with one exception: the activity in

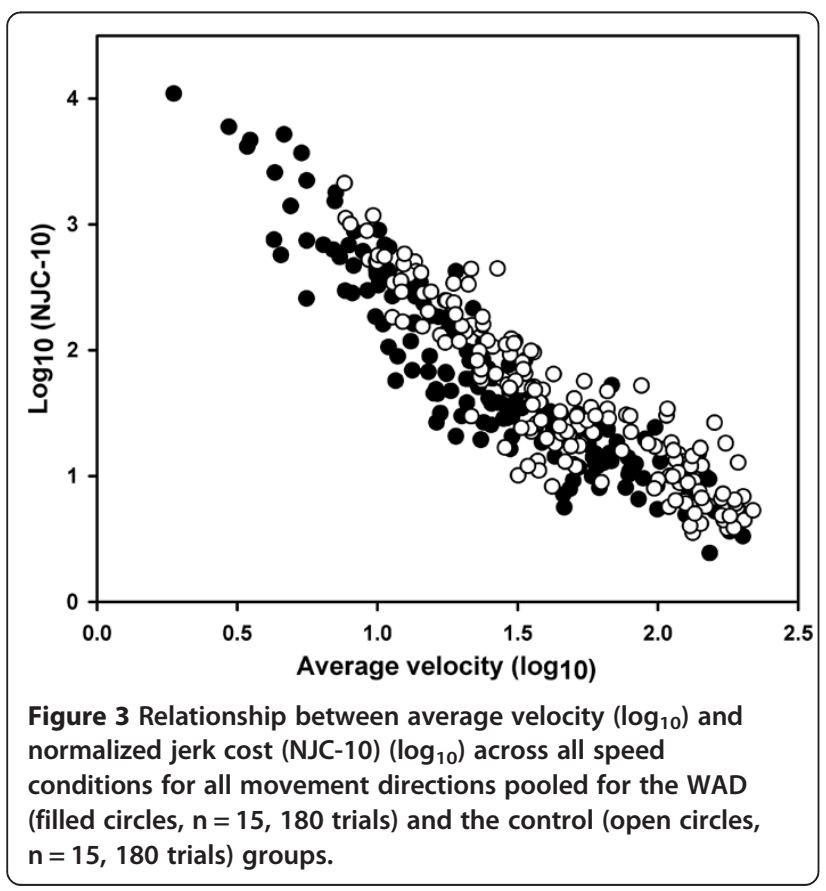

the antagonistic SCM muscle was significantly different between groups in the acceleratory phase of the EBN movement at the $\mathrm{M}$ speed condition $(\mathrm{p}<0.05)$.

\section{Association between kinematics, EMG and self-reported} data in the WAD group

We did not find any significant relationships between the self-reported pain intensity at baseline and the kinematics or rmsEMG amplitude ( $\mathrm{r}$ value range -0.44 to 0.42 , all $\mathrm{p}$-values $>0.10$ ). The NDI correlated only with the antagonistic splenius rmsEMG amplitude during the

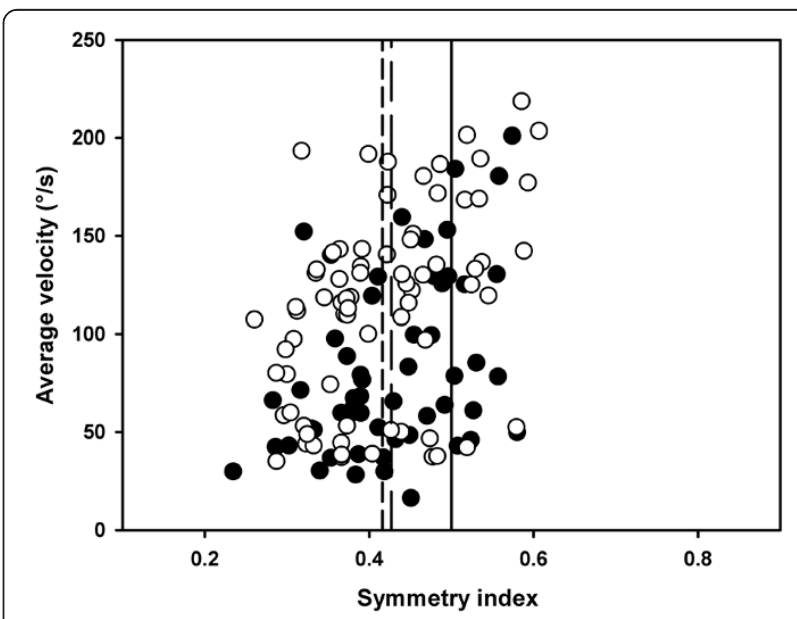

Figure 4 Velocity profile symmetry index (scale) versus average angular velocity $(\% / s)$ for the WAD (filled circles, $n=15,55$ trials) and control (open circles, $n=15,73$ trials) group. Solid vertical line indicates symmetric movements (value 0.5). Long and short dashed lines indicate the average values $(0.427 \pm 0.082$ and $0.416 \pm 0.087, p=0.81$ for group differences) for the WAD and control groups, respectively. 
Table 3 Average (SD) rms electromyographic amplitude $(\mu \mathrm{V})$ for the sternocleidomastoid (SCM) and splenius muscle at rest and at reference contractions (rest values subtracted) for the chronic WAD $(n=15)$ and control ( $n=15)$ participants

\begin{tabular}{llllll}
\hline \multirow{2}{*}{ Muscle } & \multicolumn{2}{c}{ Rest } & & \multicolumn{2}{c}{ Reference contractions } \\
\cline { 2 - 3 } \cline { 5 - 6 } & WAD & Control & & WAD & Control \\
\hline SCM & $2.75(0.26)$ & $2.74(0.19)$ & & $40.12(17.36)$ & $51.79(21.61)$ \\
Splenius & $2.73(0.69)$ & $2.52(0.17)$ & & $11.42(5.12)$ & $10.95(3.24)$ \\
\hline
\end{tabular}

There were no statistically significant differences between the groups. accelerative phase of the $\mathrm{P}$ speed at the FBN movement $(\mathrm{r}=-0.59, \mathrm{p}<0.05)$.

The FABQ physical activity subscale correlated significantly with displacement for the $\mathrm{P}$ and $\mathrm{M}$ speed condition at the EFN and FBN movement directions ( $\mathrm{r}$ value range -0.55 to -0.65 , p-values $<0.05$ ), and with peak velocity and acceleration at the $\mathrm{M}$ speed condition for the EFN and FBN movements ( $\mathrm{r}$ value range -0.52 to -0.59 , p-values $<0.05$ ).

For the FBN movement the FABQ physical activity subscale correlated with the rmsEMG amplitude for the agonist muscle at both the accelerative and decelerative phase for the $\mathrm{P}$ and $\mathrm{M}$ speed tests ( $\mathrm{r}$ value range -0.67 to -0.77 , p-values $<0.01$ ).

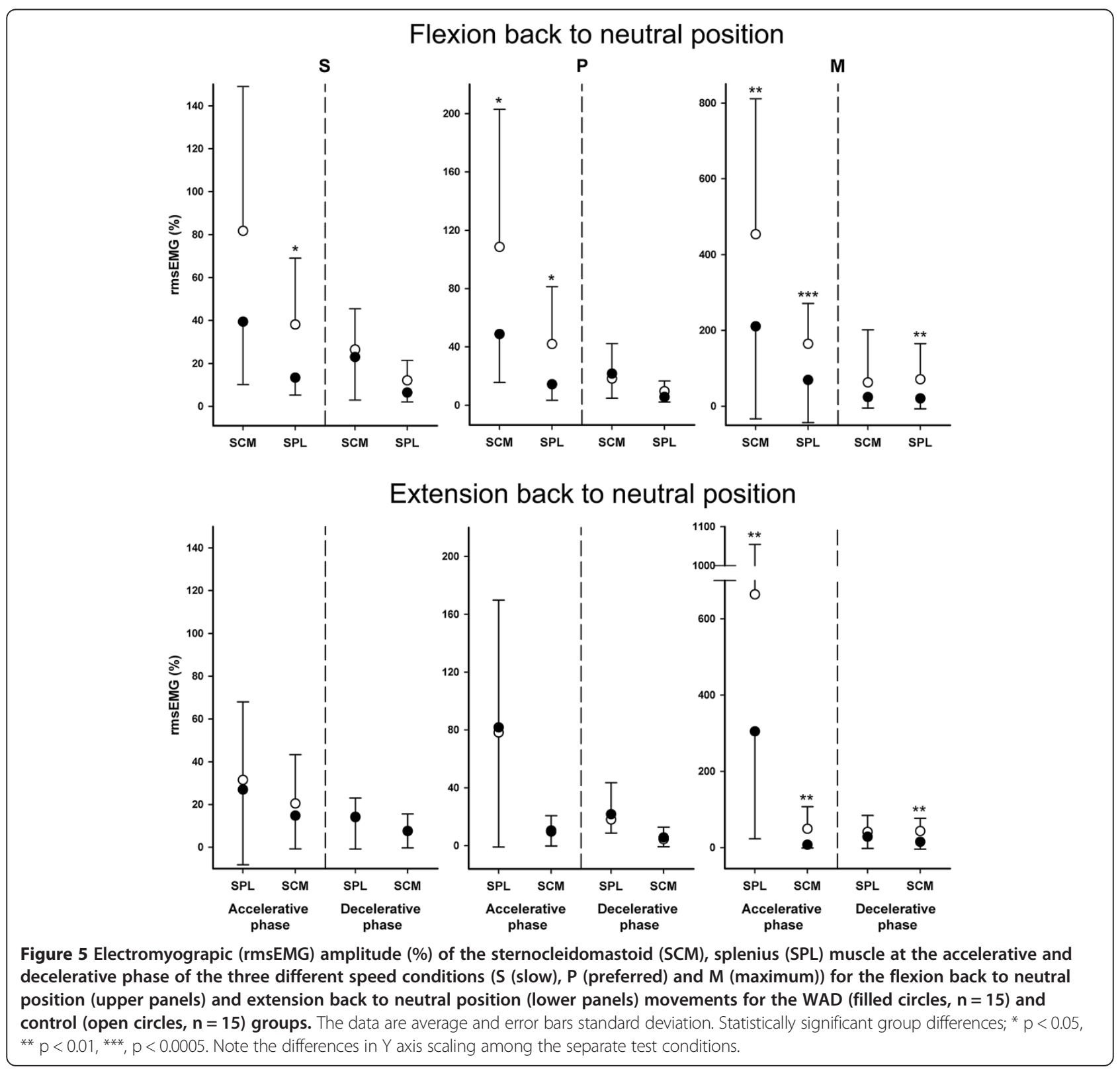




\section{Supplementary data}

Participant groups were additionally examined using extra loading (+25\% of head mass) at the $\mathrm{P}$ speed test only. The results for each group were similar to that of the unloaded P speed condition and the data are therefore shown in the Additional file 1.

\section{Discussion and conclusions}

The findings in the present study of generally reduced displacement, peak acceleration, deceleration and velocity at the maximum (M) speed conditions for the WAD group compared to controls are in close agreement with a number of previous studies examining participants with chronic WAD [10-17]. For the EFN and FBN movements we also found reduced displacement, peak acceleration, deceleration and velocity at the preferred (P) movement speed to be different between groups, which are consistent with previous observations [14]. Thus, even though the WAD participants held a large reserve capacity in movement velocity as displayed by the result of the $M$ speed test, they preferred to move at a lower velocity than the controls for the P speed test. Differences in movement velocity and displacement at both the maximum and preferred speed between the participants with chronic WAD and controls therefore seem to be a general and robust finding. The pain level of the WAD group increased significantly following the experiment, which seems to be a common finding in studies involving physical exertion of various intensity by persons with musculoskeletal disorders [40,41].

The lower peak velocity and acceleration at the $M$ speed condition for the WAD group compared with the controls also persisted after controlling for movement displacement. There are several possible explanations for such a group difference including muscle morphological and muscle activation strategies. Since we neither measured single cell- nor gross muscle area in the present study, we cannot exclude muscle atrophy as a possible factor for reductions in peak acceleration or velocity. However, when using magnetic resonance imaging, previous studies have not detected atrophy of the total cervical muscle cross-sectional area in chronic WAD participants area as assessed by case-control studies [42-44] or in a 6-month follow-up study of WAD participants [45]. Thus, group differences in neck muscle size seem to have limited explanatory strength for differences in head kinematics in the present study. As the maximum shortening velocity of a muscle is strongly dependent upon its fiber type composition [46], an increase in the proportion of slow muscle fibers of the neck muscles could possibly reduce the head movement velocity. However, the result from an uncontrolled, cross-sectional study of participants with neck pain of various etiologies on the contrary indicates a possible, minor increase in the fast fiber type direction [47]. Also, the reported type 1 fiber proportion in the neck muscles from the participants with post-traumatic etiology in the study of Uhlig [47] is almost identical to that found in presumably healthy participants [48]. Thus, it seems that the most reasonable explanation for the altered kinematics in the WAD participants would be the muscle activation patterns. We found a large reduction in agonist rmsEMG amplitude at the $M$ speed test in the WAD group compared with the controls, which supports the interpretation that the reductions in peak acceleration and velocity at the $M$ speed tests are a result of lowered muscle activation. This reduced activation found in the $M$ speed test may in turn be partly explained by fear of pain since we found significant negative associations between the FABQ physical activity component and both peak acceleration, velocity and agonist muscle rmsEMG amplitude in the $M$ speed test. Moderate relationships between fear-avoidance beliefs and displacement [49] or force [50] have also been reported previously in subjects with neck pain. It is therefore possible that peak exertion is voluntarily reduced to sub-maximal levels partly because of pain and/or fear of pain. It is also possible that the peak muscle activation is reduced because of motor inhibition by pain afferents [51,52]. The reduction in neural drive at the $M$ speed test may also be a function of both sub-maximal voluntary activation and motor inhibition.

The electromyographic activity of the agonist and antagonist muscles during both the acceleratory and deceleratory phases of the movement was on the other hand not different between groups when movement velocity and displacement were taken into consideration. Thus, the results of the present study suggest that for a given velocity and displacement of dynamic neck movements, the chronic WAD participants activated the involved muscles to the same degree as healthy controls. Although we are not aware of any studies that have examined neck muscle activation during dynamic unconstrained neck movements in WAD participants, one study examining participants with chronic non-specific neck pain and controls reported no group difference in EMG amplitude of the cervical erector spinae muscles at a durationcontrolled EBN movement [53]. These data are in keeping with our data, but they contrast somewhat with previous trials using isometric contractions [5,18]. For example, Schomacher [5] found the average EMG activity $(\mu \mathrm{V})$ of the semispinalis muscle to be significantly lower in participants with chronic WAD compared with controls during circular isometric contractions at standard force levels $(15$ and $30 \mathrm{~N})$. Their data strongly indicate that other neck muscles, either synergistic or antagonistic, must have altered their activity in parallel with that of semispinalis to generate the resultant forces. There were no indications of such a rearrangement of intermuscular activation patterns 
in the present study since we found no difference in the rmsEMG amplitude between groups after controlling for velocity and displacement for either the splenius or the SCM muscles in any movement direction. It is possible that such differences may be attributed to the muscle contraction types examined and/or the relative voluntary effort used in tests.

The smoothness and regularity of movement did not differ between groups after the movements were controlled for velocity and displacement. As indicated in Figure 3, the WAD group in fact tended to move more smoothly for a given velocity than the controls. This contrast between groups can however be explained by differences in movement displacement, since a movement of a given velocity becomes smoother by reductions in displacement [23] and this has not been taken into account in the figure. Thus, across a large range of head movement velocity, chronic neck pain due to WAD does not seem to alter the smoothness of movements compared with controls. This finding therefore contradicts the conclusions drawn from previous studies that did not control for movement velocity that implicitly suggested that movement smoothness in unconstrained movements is altered per se for participants with chronic neck pain $[11,15,19,21]$. Also, to further assess the dynamic movement strategies, we also examined the symmetry of movements and the spatial occurrence of submovements and found no significant group differences. These findings indicate that chronic WAD neither lead to a rearrangement of intermuscular activation patterns nor resultant movement patterns per se in relatively simple, unconstrained dynamic head movements. This conclusion is also somewhat in contrast to other studies that have found increased irregularity of velocitycontrolled and constrained motion paths in chronic WAD compared with healthy participants [54,55]. The head movements used in both these studies were highly spatially constrained by the imposition of visual trajectory tracking [54,55]. As several [56-59], although not all [60] studies have found reduced eye-movement control in chronic WAD, it is possible that the different conclusions made may be related to the dependence on visual involvement in the movement tests used.

A key question relates to the external validity of the study. Are the two groups of participants comparable for variables not related to neck pain? And are the chronic WAD participants representative for patients with chronic WAD group I and II? While there were no group differences with respect to descriptive data for age, anthropometrics or grip strength, the WAD group scored significantly poorer than the controls for both the physical and mental component summary scales of the SF-36, reflecting limitations in physical ability and psychological distress. Such reductions in scores of SF-36 seem to be a common finding in people with chronic musculoskeletal diseases [61,62]. The control group scored about the same as the Norwegian normative values for both the physical and mental component summary scales of the SF-36. The WAD group displayed moderate physical disability due to neck pain as measured by the NDI. The mean score for the NDI are comparable to the participants with chronic WAD grade I-II in a series of studies $[4,7,43,62,63]$, all displaying absolute NDI values very similar to our study (20-25.6). The findings in the present study should be treated with some caution due to the limited number of observations in this study. However, despite the moderate sample size, we found a number of statistically significant group differences. These findings were also seen across four different movements which further strengthens the findings.

\section{Conclusion and clinical implications}

During simple, relatively unconstrained head movements, participants with chronic WAD move with less velocity and displacement compared with healthy controls. When taking these variables into consideration we found no difference in either rmsEMG amplitude or movement smoothness between the groups. People with chronic WAD do not seem to display signs of altered motor control patterns during unconstrained dynamic head movements. We suggest that while reductions in movement velocity and displacement are robust changes and may be of clinical importance in chronic WAD, movement smoothness of unconstrained dynamic movements is not.

\section{Additional files}

Additional file 1: Data for movements at preferred speed with an additional load of $25 \%$ of head mass.

Additional file 2: Methodological description.

\section{Abbreviations}

A.u.: Arbitrary units; EBN: Extension back to the neutral head position; EFN: Extension from the neutral head position; FABQ: Fear avoidance beliefs questionnaire; FBN: Flexion back to the neutral head position; FFN: Forward flexion from the neutral head position; M: Maximum movement speed; NDI: Neck disability index; NJC: Normalized jerk cost; NP: Neutral head position; P: Preferred movement speed; rmsEMG: Root mean square electromyography; S: Slow movement speed; SCM: Sternocleidomastoid; SF-36: Short form-36; WAD: Whiplash associated disorders.

\section{Competing interests}

The authors declare that they have no competing interests.

\section{Authors' contributions}

HV designed the study, processed the data, performed statistical analyses, drafted and revised the manuscript and participated in data sampling. ESB sampled the data, and participated in the design of the study and in the drafting of the manuscript. KL performed statistical analyses, participated in data processing and revised the manuscript. SRE sampled the data and participated in drafting the manuscript. NKV designed the study, participated 
in data processing and revised the manuscript. All authors read and approved the final manuscript.

\section{Acknowledgements}

The authors are grateful to all participants in this study. We would also like to thank Bredo Knudtzen, Trygve F. Moe, Hildegun Snekkevik, John Vikne, Arit Ødegaard and Kjell Øygard for their helpfulness and support of the present study. The first author was financially supported by the Norwegian ExtraFoundation for Health and Rehabilitation through EXTRA funds.

\section{Author details}

1Department of Health Sciences, Institute of Health and Society, University of Oslo, P.O. Box 1089, Blindern, NO-0317 Oslo, Norway. Department of Informatics, University of Oslo, P.O. Box 1080, Blindern, NO-0316 Oslo, Norway.

\section{Received: 25 February 2013 Accepted: 22 October 2013}

Published: 4 November 2013

\section{References}

1. Soderlund A, Lindberg P: Long-term functional and psychological problems in whiplash associated disorders. Int J Rehabil Res 1999, 22(2):77-84.

2. Sterling M, Jull G, Vicenzino B, Kenardy J, Darnell R: Development of motor system dysfunction following whiplash injury. Pain 2003, 103(1-2):65-73.

3. Barton PM, Hayes KC: Neck flexor muscle strength, efficiency, and relaxation times in normal subjects and subjects with unilateral neck pain and headache. Arch Phys Med Rehabil 1996, 77(7):680-687.

4. Pearson I, Reichert A, De Serres SJ, Dumas JP, Cote JN: Maximal voluntary isometric neck strength deficits in adults with whiplash-associated disorders and association with pain and fear of movement. $J$ Orthop Sports Phys Ther 2009, 39(3):179-187.

5. Schomacher J, Farina D, Lindstroem R, Falla D: Chronic trauma-induced neck pain impairs the neural control of the deep semispinalis cervicis muscle. Clin Neurophysio/ 2012, 123(7):1403-1408.

6. Dumas JP, Arsenault AB, Boudreau G, Magnoux E, Lepage Y, Bellavance A, Loisel P: Physical impairments in cervicogenic headache: traumatic vs. nontraumatic onset. Cephalalgia 2001, 21(9):884-893.

7. Kumbhare DA, Balsor B, Parkinson WL, Harding Bsckin P, Bedard M, Papaioannou A, Adachi JD: Measurement of cervical flexor endurance following whiplash. Disabil Rehabil 2005, 27(14):801-807.

8. Larsson SE, Alund M, Cai H, Oberg PA: Chronic pain after soft-tissue injury of the cervical spine: trapezius muscle blood flow and electromyography at static loads and fatigue. Pain 1994, 57(2):173-180

9. Woodhouse A, Liljeback P, Vasseljen O: Reduced head steadiness in whiplash compared with non-traumatic neck pain. J Rehabil Med 2010, 42(1):35-41.

10. Ohberg F, Grip H, Wiklund U, Sterner Y, Karlsson JS, Gerdle B: Chronic whiplash associated disorders and neck movement measurements: an instantaneous helical axis approach. IEEE Trans Inf Technol Biomed 2003, 7(4):274-282.

11. Grip H, Sundelin G, Gerdle B, Karlsson JS: Cervical helical axis characteristics and its center of rotation during active head and upper arm movementscomparisons of whiplash-associated disorders, non-specific neck pain and asymptomatic individuals. J Biomech 2008, 41(13):2799-2805

12. Dall'Alba PT, Sterling MM, Treleaven JM, Edwards SL, Jull GA: Cervical range of motion discriminates between asymptomatic persons and those with whiplash. Spine 2001, 26(19):2090-2094.

13. Armstrong BS, McNair PJ, Williams M: Head and neck position sense in whiplash patients and healthy individuals and the effect of the craniocervical flexion action. Clin Biomech 2005, 20(7):675-684.

14. Baydal-Bertomeu JM, Page AF, Belda-Lois JM, Garrido-Jaen D, Prat JM: Neck motion patterns in whiplash-associated disorders: quantifying variability and spontaneity of movement. Clin Biomech 2011, 26(1):29-34.

15. Feipel V, Rondelet B, LePallec JP, DeWitte O, Rooze M: The use of disharmonic motion curves in problems of the cervical spine. Int Orthop 1999, 23(4):205-209.

16. Pereira MJ, Jull GA, Treleaven JM: Self-reported driving habits in subjects with persistent whiplash-associated disorder: relationship to sensorimotor and psychologic features. Arch Phys Med Rehabil 2008, 89(6):1097-1102.

17. Madeleine $P$, Prietzel $H$, Svarrer $H$, Arendt-Nielsen L: Quantitative posturography in altered sensory conditions: a way to assess balance instability in patients with chronic whiplash injury. Arch Phys Med Rehabil 2004 85(3):432-438
18. Jull G, Kristjansson E, Dall'Alba P: Impairment in the cervical flexors: a comparison of whiplash and insidious onset neck pain patients. Man Ther 2004, 9(2):89-94.

19. Sjölander $P$, Michaelson $P$, Jaric S, Djupsjöbacka M: Sensorimotor disturbances in chronic neck pain-range of motion, peak velocity, smoothness of movement, and repositioning acuity. Man Ther 2008, 13(2):122-131.

20. Freund $\mathrm{HJ}$, Budingen $\mathrm{HJ}$ : The relationship between speed and amplitude of the fastest voluntary contractions of human arm muscles. Exp Brain Res 1978, 31(1):1-12

21. Sarig Bahat $H$, Weiss $P L$, Laufer $Y$ : The effect of neck pain on cervical kinematics, as assessed in a virtual environment. Arch Phys Med Rehabil 2010, 91(12):1884-1890.

22. Hogan N, Flash T: Moving gracefully - quantitative theories of motor coordination. Trends Neurosci 1987, 10(4):170-174.

23. Vikne H, Bakke ES, Liestøl K, Sandbæk G, Vøllestad N: The smoothness of unconstrained head movements is velocity-dependent. Hum Mov Sci 2013, 32(4):540-554

24. Spitzer WO, Skovron ML, Salmi LR, Cassidy JD, Duranceau J, Suissa S, Zeiss E: Scientific monograph of the quebec task force on whiplash-associated disorders: redefining "whiplash" and its management. Spine 1995, 20(8 Suppl):1S-73S.

25. McConville JT, Chuchill TD, Kaleps I, Clauser CE Cuzzi J: Anthropometric relationships of body and body segment moments of inertia. Dayton, Ohio: Aerospace Medical Research Laboratory, Wright-Patterson Air Force Base; 1980.

26. Young JW, Chandler RF, Snow CC, Robinette KM, Zehner GF, Lofberg MS: Anthropometric and mass distribution characteristics of the adults female Oklahoma: FAA Civil Aeromedical Institute Oklaoma City; 1983.

27. Chandler RF, Clauser CE, McConville JT, Reynolds HM, Young JW: Investigation of inertial properties of the human body. Washington: U.S. Department of Transportation, 1975.

28. Wind $A E$, Takken $T$, Helders PJ, Engelbert RH: Is grip strength a predictor for total muscle strength in healthy children, adolescents, and young adults? Eur J Pediatr 2010, 169(3):281-287.

29. Wang $M$, Leger $A B$, Dumas GA: Prediction of back strength using anthropometric and strength measurements in healthy females. Clin Biomech 2005, 20(7):685-692

30. Vernon H, Mior S: The Neck Disability Index: a study of reliability and validity. J Manipulative Physiol Ther 1991, 14(7):409-415.

31. Waddell G, Newton M, Henderson I, Somerville D, Main CJ: A fearavoidance beliefs questionnaire (FABQ) and the role of fear-avoidance beliefs in chronic low back pain and disability. Pain 1993, 52(2):157-168.

32. Cleland JA, Fritz JM, Childs JD: Psychometric properties of the fearavoidance beliefs questionnaire and tampa scale of kinesiophobia in patients with neck pain. Am J Phys Med Rehabil 2008, 87(2):109-117.

33. Ware JE, Snow KK, Kosinski MA, Gandek B: SF-36 health survey manual and interpretation quide. Boston, MA: The Health Institute, New England Medical Center; 1993.

34. Loge $J H$, Kaasa S: Short form 36 (SF-36) health survey: normative data from the general norwegian population. Scand J Soc Med 1998, 26(4):250-258.

35. Teulings $\mathrm{HL}$, Contreras-Vidal $\mathrm{J}$, Stelmach $\mathrm{GE}$, Adler $\mathrm{CH}$ : Parkinsonism reduces coordination of fingers, wrist, and arm in fine motor control. Exp Neurol 1997, 146(1):159-170.

36. Ketcham CJ, Seidler RD, Van Gemmert AW, Stelmach GE: Age-related kinematic differences as influenced by task difficulty, target size, and movement amplitude. J Gerontol B Psychol Sci Soc Sci 2002, 57(1):54-64

37. Nagasaki $\mathrm{H}$ : Asymmetric velocity and acceleration profiles of human arm movements. Exp Brain Res 1989, 74(2):319-326.

38. Falla D, Dall'Alba P, Rainoldi A, Merletti R, Jull G: Location of innervation zones of sternocleidomastoid and scalene muscles-a basis for clinical and research electromyography applications. Clin Neurophysiol 2002, 113:57-63.

39. Mustard BE, Lee RG: Relationship between EMG patterns and kinematic properties for flexion movements at the human wrist. Exp Brain Res 1987, 66(2):247-256

40. Roe C, Brox Jl, Saugen E, Vollestad NK: Muscle activation in the contralateral passive shoulder during isometric shoulder abduction in patients with unilateral shoulder pain. J Electromyogr Kinesio/ 2000, 10(2):69-77.

41. Andersen LL, Nielsen PK, Sogaard K, Andersen CH, Skotte J, Sjogaard G Torque-EMG-velocity relationship in female workers with chronic neck muscle pain. J Biomech 2008, 41(9):2029-2035.

42. Matsumoto M, Ichihara D, Okada E, Chiba K, Toyama Y, Fujiwara H, Momoshima S, Nishiwaki Y, Takahata T: Cross-sectional area of the posterior extensor muscles of the cervical spine in whiplash injury 
patients versus healthy volunteers-10 year follow-up MR study. Injury 2012, 43(6):912-916.

43. Elliott J, Jull G, Noteboom JT, Galloway G: MRI study of the cross-sectional area for the cervical extensor musculature in patients with persistent whiplash associated disorders (WAD). Man Ther 2008, 13(3):258-265.

44. Elliott JM, O'Leary S, Sterling M, Hendrikz J, Pedler A, Jull G: Magnetic resonance imaging findings of fatty infiltrate in the cervical flexors in chronic whiplash. Spine 2010, 35(9):948-954.

45. Ulbrich EJ, Aeberhard R, Wetli S, Busato A, Boesch C, Zimmermann H, Hodler J, Anderson SE, Sturzenegger M: Cervical muscle area measurements in whiplash patients: acute, 3 , and 6 months of followup. J Magn Reson Imaging 2012, 36(6):1413-1420.

46. Schiaffino $S$, Reggiani C: Fiber types in mammalian skeletal muscles. Physiol Rev 2011, 91(4):1447-1531.

47. Uhlig Y, Weber BR, Grob D, Muntener M: Fiber composition and fiber transformations in neck muscles of patients with dysfunction of the cervical spine. J Orthop Res 1995, 13(2):240-249.

48. Vikne H, Gundersen K, Liestol K, Maelen J, Vollestad N: Intermuscular relationship of human muscle fiber type proportions: slow leg muscles predict slow neck muscles. Muscle Nerve 2012, 45(4):527-535.

49. Pedler A, Sterling M: Assessing fear-avoidance beliefs in patients with whiplash-associated disorders: a comparison of 2 measures. Clin J Pain 2011, 27(6):502-507.

50. Lindstroem R, Graven-Nielsen T, Falla D: Current pain and fear of pain contribute to reduced maximum voluntary contraction of neck muscles in patients with chronic neck pain. Arch Phys Med Rehabil 2012, 93(11):2042-2048.

51. Graven-Nielsen T, Lund H, Arendt-Nielsen L, Danneskiold-Samsoe B, Bliddal H: Inhibition of maximal voluntary contraction force by experimental muscle pain: a centrally mediated mechanism. Muscle Nerve 2002, 26(5):708-712.

52. Graven-Nielsen T, Svensson P, Arendt-Nielsen L: Effects of experimental muscle pain on muscle activity and co-ordination during static and dynamic motor function. Electroencephalogr Clin Neurophysiol 1997, 105(2):156-164.

53. Maroufi N, Ahmadi A, Mousavi Khatir SR: A comparative investigation of flexion relaxation phenomenon in healthy and chronic neck pain subjects. Eur Spine J 2013, 22(1):162-168.

54. Kristjansson E, Hardardottir L, Asmundardottir M, Gudmundsson K: A new clinical test for cervicocephalic kinesthetic sensibility: "the fly". Arch Phys Med Rehabil 2004, 85(3):490-495.

55. Woodhouse A, Stavdahl O, Vasseljen O: Irregular head movement patterns in whiplash patients during a trajectory task. Exp Brain Res 2010, 201(2):261-270.

56. Gimse R, Tjell C, Bjorgen IA, Saunte C: Disturbed eye movements after whiplash due to injuries to the posture control system. J Clin Exp Neuropsychol 1996, 18(2):178-186.

57. Treleaven J, Jull G, Grip H: Head eye co-ordination and gaze stability in subjects with persistent whiplash associated disorders. Man Ther 2011, 16(3):252-257.

58. Tjell C, Rosenhall U: Smooth pursuit neck torsion test: a specific test for cervical dizziness. Am J Otol 1998, 19(1):76-81.

59. Treleaven J, Jull G, LowChoy N: Smooth pursuit neck torsion test in whiplash-associated disorders: relationship to self-reports of neck pain and disability, dizziness and anxiety. J Rehabil Med 2005, 37(4):219-223.

60. Kongsted A, Jorgensen LV, Bendix T, Korsholm L, Leboeuf-Yde C: Are smooth pursuit eye movements altered in chronic whiplash-associated disorders? A cross-sectional study. Clin Rehabil 2007, 21(11):1038-1049.

61. Boonstra AM, Reneman MF, Stewart RE, Post MW, Schiphorst Preuper HR Life satisfaction in patients with chronic musculoskeletal pain and its predictors. Qual Life Res 2013, 22(1):93-101.

62. Juul-Kristensen B, Clausen B, Ris I, Jensen RV, Steffensen RF, Chreiteh SS, Jorgensen MB, Sogaard K: Increased neck muscle activity and impaired balance among females with whiplash-related chronic neck pain: a cross-sectional study. J Rehabil Med 2013, 45(4):376-384.

63. Falla D, Bilenkij G, Jull G: Patients with chronic neck pain demonstrate altered patterns of muscle activation during performance of a functional upper limb task. Spine 2004, 29(13):1436-1440.

\section{doi:10.1186/1471-2474-14-314}

Cite this article as: Vikne et al:: Muscle activity and head kinematics in unconstrained movements in subjects with chronic neck pain; cervical motor dysfunction or low exertion motor output? BMC Musculoskeletal Disorders 2013 14:314.

\section{Submit your next manuscript to BioMed Central and take full advantage of:}

- Convenient online submission

- Thorough peer review

- No space constraints or color figure charges

- Immediate publication on acceptance

- Inclusion in PubMed, CAS, Scopus and Google Scholar

- Research which is freely available for redistribution 